\title{
A necropsy and histomorphometric study of abnormalities in the course of the vertebral artery associated with ossified stylohyoid ligaments
}

C P Johnson, M Scraggs, T How, J Burns

\begin{abstract}
Aims-To establish whether abnormalities in the course of the vertebral artery occur and whether they are relevant to arterial injury associated with head and neck movements.
\end{abstract}

Methods-Twenty vertebral arteries were carefully dissected at necropsy and abnormalities in course were noted, along with any other bony or cartilaginous cervical anomalies. The effect of head and neck movement on these vessels was studied before a detailed histomorphometric examination was undertaken on sections of the excised arteries.

Results-Five vessels had an abnormal course. One vessel entered the transverse foramina of the fifth cervical vertebra rather than the sixth, but was otherwise normal. In two subjects both vertebral arteries were abnormal in the upper cervical portion with, in each case, a straight left vertebral artery and a right vertebral artery with a deficient loop, closely applied to the atlanto-axial joint. Both of these subjects also had completely ossified stylohyoid ligaments and the arteries visibly stretched with modest head and neck movements. Histology revealed variable degrees of smooth muscle disarray in the tunica media of two of the arteries with loop deficiencies. The circumference of one of the straight arteries was smaller than expected but in all other measured histomorphometric parameters these vessels appeared normal.

Conclusions-Vertebral artery loops are deficient in a number of subjects. This finding is important given the recently described biomechanical susceptibility of the vertebral artery to longitudinal extension and may explain the smooth muscle changes, in that this may represent attempts at arterial wall remodelling. Subjects with such loop deficiencies may be more susceptible to a variety of head and neck insults and such abnormalities should be sought at necropsy in subjects who die as a result of fatal vertebral artery injury. ( $\mathcal{H}$ Clin Pathol 1995;48:637-640)

Keywords: Vertebral artery, abnormalities, injury, neck movement.
Vertebral artery injuries pose a number of difficulties for those involved with the diagnosis, treatment and prevention of these events, which often lead to serious neurological disability or death. This is particularly important because the subjects are often relatively young and apparently fit before the precipitating event. The mechanism of arterial injury is probably multifactorial but there is increasing interest in the role of rotational movements around the atlanto-axial joint, particularly in those cases associated with therapeutic neck manipulation. The evidence for this has been reviewed by Thiel. ${ }^{1}$ One of the key problems with this concept is that only a very small proportion of people who undergo such rotational movements will experience any adverse effects. In the cases that have been examined radiologically or at necropsy intimal tearing with intraluminal thrombosis or dissecting aneurysm seems to be responsible for causing the decrease in posterior cerebral perfusion. ${ }^{2-4}$ Some of these subjects certainly have arterial

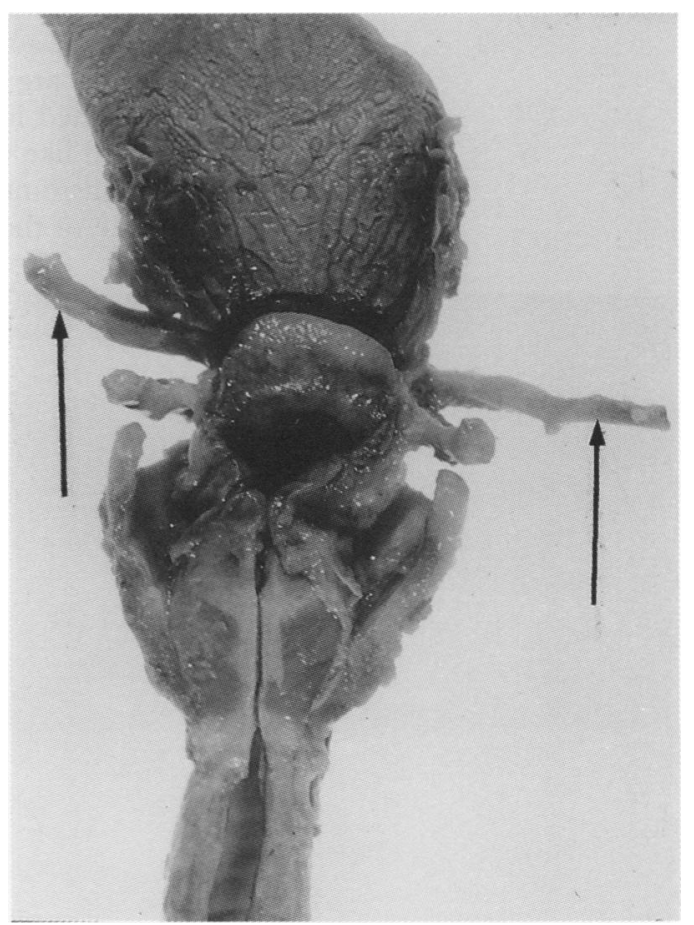

Figure 1 A posterior view of the larynx showing part of the ossified stylohyoid ligaments (arrows), which were in continuity with the base of the skull (case 1). 


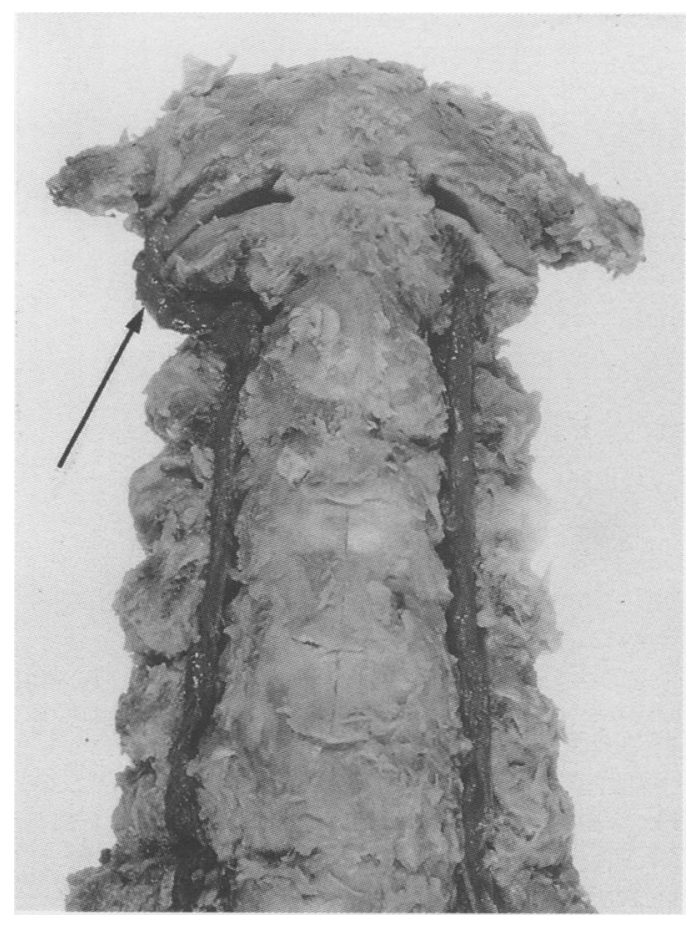

Figure 2 Frontal view of the cervical spine following exposure of the vertebral arteries (highlighted with artist's ink). Note the straight left vertebral artery and the small right-sided loop, closely apposed to the atlanto-axial joint (arrow). The neck specimen has been removed from the cadaver to improve the photography (case 1).

wall abnormalities such as medial cystic necrosis $^{5}$ and the more serious sequelae appear more likely if the collateral circulation is impaired by contralateral arterial hypoplasia. Similar rotational movements may occur during physical assaults to the side of the head and neck, either from the blow itself or, as suggested by $\mathrm{Gee}^{6}{ }^{6}$ in attempts to avoid such an impact, possibly contributing to the subsequent vertebral artery rupture. The vertebral artery loops, in the proximal cervical portion, are thought to provide adequate protection against such injuries, an argument which we feel has not been examined in any detail. With this in mind, we would like to present the results of a necropsy and histomorphometric study which addresses some of these points.

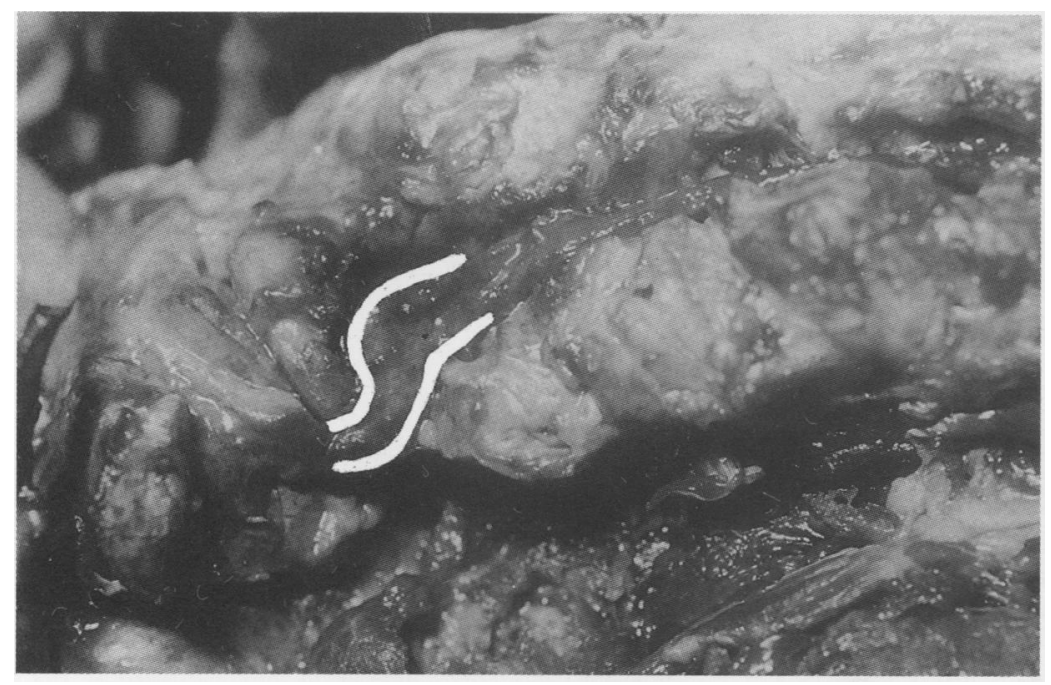

Figure 3 The right side of the cervical spine, photographed in situ, showing the small right-sided loop. Note the short length of the segment (highlighted in white; case 1).

\section{Methods}

Twenty fresh vertebral arteries, from 17 subjects who had died of non-cerebral disease, were carefully examined following dissection at necropsy, as previously described, ${ }^{7}$ as part of an ongoing biomechanical and histomorphometric study of the vertebral artery.

Abnormalities in the origin and course of the extracranial portion of the vessels were noted, along with any associated osseous, or cartilaginous, anomalies in the neck. The effect on the extracranial artery of moving the head and neck of the cadaver was observed before removal of the vessel from the body. Formaldehyde fixed tissue from along these arteries was processed routinely and representative histological sections were stained with haematoxylin and eosin, alcian blue ( $\mathrm{pH} \mathrm{2.5)}$ and Miller's elastin van Gieson. These sections were carefully studied using light microscopy before a detailed histomorphometric examination was undertaken using a Seescan image analyser. This allowed the average circumference, wall thickness, medial thickness, adventitial:medial area ratio, and the intimal:medial area ratio for each vessel to be assessed. An estimation of proteoglycan accumulation in the tunica media (as assessed by alcian blue staining) was undertaken, as previously described, ${ }^{8}$ using an eyepiece graticule.

\section{Results}

All of the arteries had a normal origin from the subclavian artery. Fifteen vessels had a normal course, entering the bony canal at the transverse process of the sixth cervical vertebra, looping around the atlas before piercing the membrane and the dura to enter the posterior cranial fossa. Although there was some variation in the shape and size of these loops, all appeared to take the course of the vertebral artery away from the atlanto-axial joint, so that when modest rotational and extensional neck movements were applied, the loops either partially or completely straightened out, but did not appear to stretch visibly. One artery entered the bony canal at the fifth cervical vertebra but otherwise had a normal course. In two subjects both arteries had noticeably abnormal courses at the atlantoaxial level. A 65 year old woman (case 1) had complete ossification of both stylohyoid ligaments (fig 1) associated with total absence of a left vertebral artery loop and an abnormally small loop of the right vertebral artery, with this artery lying directly on the atlantoaxial joint (fig 2). The lateral view of this artery (fig 3) illustrates the particularly short anterioposterior course at this level. A 33 year old woman (case 2) had complete ossification of the left stylohyoid ligament, also lacked a loop on this side and, again, had a deficient loop of the right artery lying on the atlanto-axial joint. In both of these cadavers modest rotational and extensional neck movements led to obvious stretching of the arteries in the upper cervical portion. Light microscopic examination of the left vertebral artery from case 1 revealed a very unusual arrangement of the smooth muscle cells in the tunica media. Instead of the normal 
Results (mean $\pm S D$ ) of histomorphometric analysis of the upper cervical portions of the 16 vessels with normal loops and the four vessels with loop deficiencies

\begin{tabular}{|c|c|c|c|c|c|}
\hline & Circumference (mm) & Wall thickness (mm) & Medial width $(\mathrm{mm})$ & A:M ratio & I:M ratio \\
\hline $\begin{array}{l}\text { Normal arteries }(n=16) \\
\text { Case no. } 1\end{array}$ & $11 \cdot 51 \pm 2 \cdot 12$ & $0.395 \pm 0.09$ & $0.232 \pm-0.06$ & $0.496 \pm 0.19$ & $0 \cdot 261 \pm 0.21$ \\
\hline $\begin{array}{l}\text { left } \\
\text { right }\end{array}$ & $\begin{array}{l}8 \cdot 64 \pm 0.39 * \\
10 \cdot 56 \pm 0.8\end{array}$ & $\begin{array}{l}0 \cdot 468 \pm 0 \cdot 12 \\
0 \cdot 446 \pm 0 \cdot 10\end{array}$ & $\begin{array}{l}0.226 \pm 0.06 \\
0 \cdot 300 \pm 0.07\end{array}$ & $\begin{array}{l}0.574 \pm 0.19 \\
0.405 \pm 0.03\end{array}$ & $\begin{array}{l}0.585 \pm 0.14 \\
0.075 \pm 0.025\end{array}$ \\
\hline $\begin{array}{l}\text { Case no. } 2 \\
\text { left } \\
\text { right }\end{array}$ & $\begin{array}{c}9 \cdot 73 \pm 1.98 \\
10.56 \pm 0.8\end{array}$ & $\begin{array}{l}0.313 \pm 0.06 \\
0.353 \pm 0.03\end{array}$ & $\begin{array}{l}0 \cdot 169 \pm 0.03 \\
0 \cdot 206 \pm 0.027\end{array}$ & $\begin{array}{l}0.823 \pm 0.257 \\
0.73 \pm 0.117\end{array}$ & $\begin{array}{l}0.149 \pm 0.09 \\
0.07 \pm 0.018\end{array}$ \\
\hline
\end{tabular}

* Abnormal result. A:M ratio = adventitial:medial ratio; $\mathrm{I}: \mathrm{M}=$ intimal:medial ratio.

circumferential orientation of the muscle cells, there was noticeable disarray with the nuclei arranged in various directions (fig 4). This
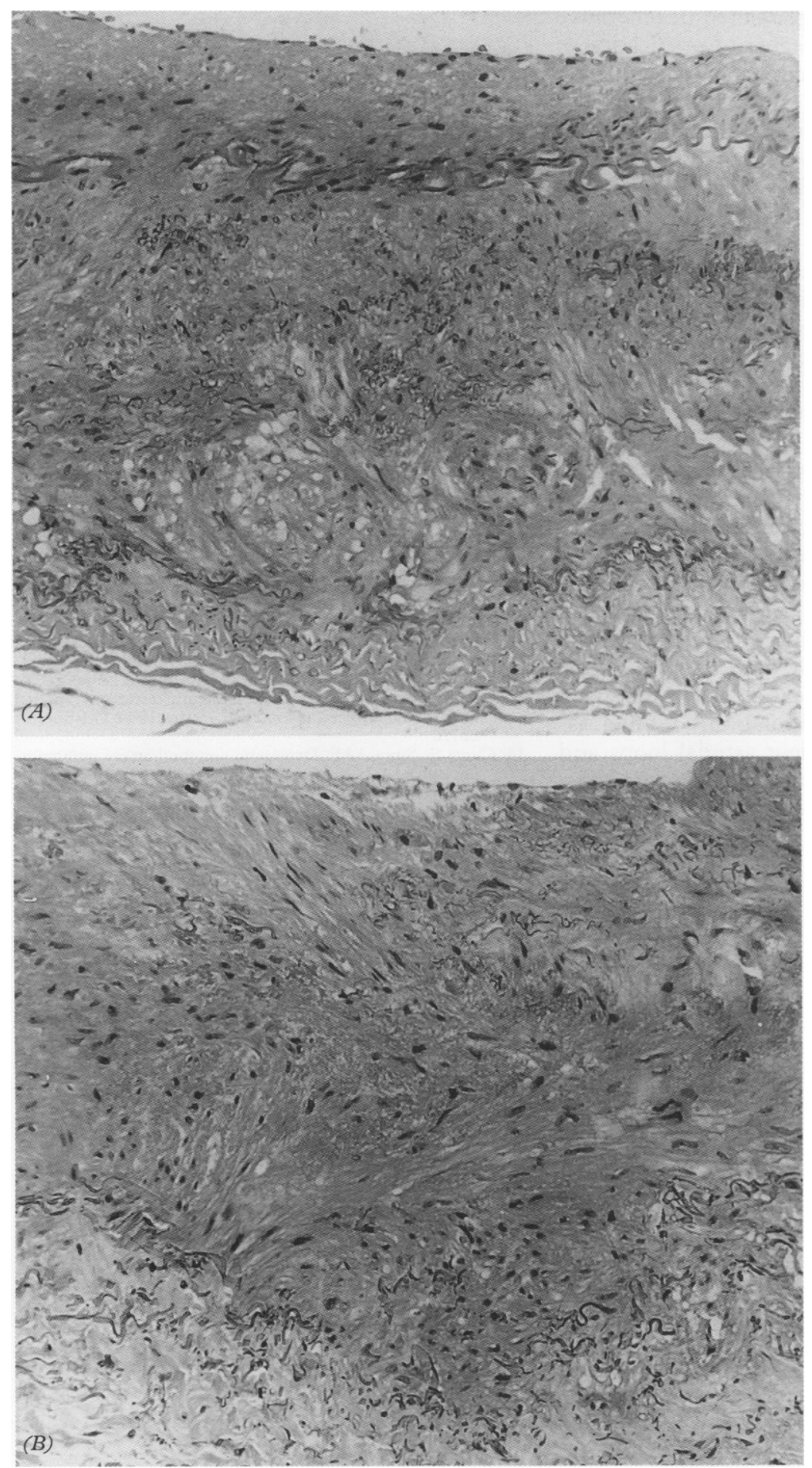

Figure 4 (A) and (B) Haematoxylin and eosin stained sections of the straight left vertebral artery of case 1 . Note the irregular and haphazard arrangement of the smooth muscle cells in the tunica media (intima towards the top of figures). change was present along the length of the vessel. Similar, but more modest, disarray was also seen in the right vertebral artery. The sections from case 2 showed mild focal disarray of the smooth muscle, probably just within the limits of normal.

The smooth muscle architecture of the other 16 vessels appeared normal except for the local disruption seen in vessels with prominent arteriosclerosis and in some arterial sections taken from the lower portions of the vertebral artery, close to the subclavian artery.

The histomorphometric data of the upper cervical portions (from the inferior border of $\mathrm{C} 1$ to the inferior border of the $\mathrm{C} 3$ vertebrae) of the 16 arteries with normal loops was compared with those of cases 1 and 2 (table). This revealed that only the circumference of the left artery of case 1 was small but that in all other measured parameters, those vessels with an abnormal course were normal. There was no measurable increase in proteoglycan accumulation in these vessels (data not shown).

\section{Discussion}

Published descriptions of vertebral artery abnormalities concentrate on asymmetry, unusual points of origin, variations in level of entry into the cervical vertebrae, and duplications, ${ }^{9-12}$ all of course important in surgical practice. We are not aware of any earlier accounts of vertebral artery loop deficiencies occurring with ossified stylohyoid ligaments. We feel that there is a lack of awareness that various abnormalities occur in this important portion of the vertebral artery, in many instances the site of supposed rotational/extensional injury. The significance of these is unclear as yet, but simple neck movements stretched these vessels at necropsy and preliminary results of our biomechanical study indicate that the vertebral artery is particularly susceptible to longitudinal extension. ${ }^{13}$ We have documented that the normal loop, which is thought to protect the artery from such forces, is deficient in a number of subjects and, along with pathological processes in the vessel wall, may explain the variable outcome in subjects experiencing unusual or sudden neck movements and, for that matter, the more overt head and neck trauma seen in forensic practice.

The histological changes seen in the smooth muscle of the tunica media may be a further manifestation of congenital abnormality; a change described as dysplasia of the muscular media has been reported in association with a noticeably hypoplastic vertebral artery. ${ }^{14}$ In subjects with deficient protective loops, how- 
ever, the smooth muscle disarray could also be explained as an attempt by these arteries to remodel in response to various forces generated by day-to-day head and neck movement. From previous work, we know that the circumference of the vertebral artery usually becomes slightly greater in the upper cervical loop and it is not entirely surprising, therefore, that a straight vertebral artery (case 1, left) does not have this feature.

It is unclear what significance the ossified stylohyoid ligaments have in relation to the abnormal course of these arteries. In this short series, all four abnormalities at the atlanto-axial level occurred in subjects with this feature. It may simply reflect an age related degenerative process, although the degree of ossification is pronounced and occurred in one subject aged 33 years. Alternatively, this could represent ossification not of a previously normal ligament, but of a persistent hyoid cartilaginous bar (second branchial arch cartilage). From the definitive work of Padget ${ }^{15}$ on the development of the cranial arteries in the human embryo, it would appear that the vertebral artery forms from fusion of the cervical intersegmental arteries during the embryological stage of development (10-15 mm embryo) when the first and second branchial arch structures predominate. One could then speculate that this association is not purely one of chance but that some problem in embryological development could account for it. Further study is obviously required to clarify and confirm this association.

We feel that these results may have both clinical and pathological implications for the study of serious neck injury. At present, there seems to be no practical and effective way to identify those individuals who may be at risk of developing neurological sequelae following therapeutic neck manipulation. If the association between deficient arterial loops and ossified stylohyoid ligaments is confirmed in a larger study, then simple cervical spine $x$ rays could prove a valuable tool for preventing some of these tragedies by enabling those individuals who may be susceptible to such injury to be identified and then advised against undergoing manipulation. Equally important, the pathologist should be aware of such abnormalities in the course and structure of the vertebral artery and consider their possible significance when investigating fatal cases of vertebral artery injury. The authors would like to thank $\mathrm{Mr}$ Alan Williams and $\mathrm{Dr}$
David Williams for photographic assistance, the anatomical pathology technical staff at the Royal Liverpool University Hospital and the Liverpool City Mortuary and Sheryl Anderson for typing the manuscript.

1 Thiel HW. Gross morphology and pathoanatomy of the vertebral arteries. $\mathcal{F}$ Manipulative Physiol Ther 1991;14: 133-41.

2 Hart RG. Vertebral artery dissection. Neurology 1988;38: 987-9.

3 Fast A, Zinicola DF, Marin EL. Vertebral artery damage complicating cervical manipulation. Spine 1987;12:840-2.

4 Sherman MR, Smialek JE, Zane WE. Pathogenesis of vertebral artery occlusion following cervical spine manipulation. Arch Pathol Lab Med 1987;111:851-3.

5 Johnson CP, Lawler W, Burns J. Histomorphometry in the assessment of fatal vertebral artery dissection. $₹$ Clin Pathol 1993;46:1000-3.

6 Gee DJ. Traumatic sub-arachnoid haemorrhage. Proceedings of the 12th Congress of the International Academy of Forensic and Social Medicine. Vienna: Egermann, 1982:495-8.

7 Bromilow A, Burns J. A technique for removal of the vertebral arteries. $f$ Clin Pathol 1985;38:1400-2.

8 Johnson CP, Burns J. The medicolegal significance of proteoglycan accumulation in the tunica media of the vertebral artery. Am $\mathcal{F}$ Forensic Med Pathol 1993;14:165-9.

9 Cavdar S, Arisan E. Variations in the extracranial origin of the human vertebral artery. Acta Anat 1989;135:236-8.

10 Takasato Y, Hayashi H, Kobayashi T, Hashimoto Y. Duplicated origin of right vertebral artery with rudimentary and accessory left vertebral arteries. Neuroradiology 1992; and access 34 : $287-9$.

11 FIynn R. External carotid origin of the dominant vertebral artery. F Neurosurg 1968;29:300-1.

12 Takenchi S, Komiya Y, Nakajima S, Hayami H. Two cases of the vertebral artery without passing through foramen transversarium of the atlas. Tokyo Med $\mathcal{F}$ 1980;95:454-61.

13 Johnson CP, How T, Scraggs M, Burns J. The poor biomechanical response of the vertebral artery to applied longitudinal stress [abstract]. $¥$ Pathol 1994;173(Suppl): 207A.

14 Curry B, Sima AAF. Dysplastic vertebral artery with paradoxical infarction. Can $\mathcal{F}$ Neurol Sci 1980;7:313-16.

15 Padget DH. The development of the cranial arteries in the human embryo. Contrib Embryol 1948;32:205-61. 